Estimation of the Electrical Parameters of a Laboratory-Scale Three-Phase Transmission Line from Transient Voltage Measurements

\title{
Estimación de los Parámetros Eléctricos de una Línea de Transmisión Trifásica a Escala de Laboratorio a Partir de Mediciones de Transitorios de Voltaje
}

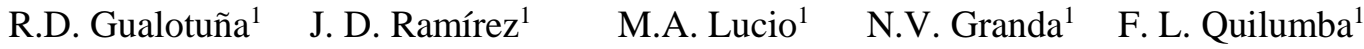 \\ ${ }^{1}$ Escuela Politécnica Nacional, Quito, Ecuador \\ E-mail: rene.gualotuna@epn.edu.ec; juan.ramirezd@epn.edu.ec; miguel.lucio@epn.edu.ec; \\ nelson.granda@epn.edu.ec; quigufrale@ieee.org
}

Abstract

This article presents the estimation of the electrical parameters of a three-phase transmission line from measurements of transient voltages in a laboratoryscale model using non-linear optimization methods. The aim of the study is to be able to determine the parameters more accurately and faster compared to conventional methods. The conventional tests open circuit and short circuit were conducted on a scale model in the Laboratory of Electrical Machines at the National Polytechnic School. The parameters derived from these tests constitute the initial electrical parameters of the model. Subsequently, a transient voltage event is simulated by energizing the line by means of a switch that provides an experimental voltage signal. The signal is captured by a recording instrument for subsequent analysis. With the help of MATLAB-Simulink, the simulation of the transmission line is performed with the respective voltage transient obtained by energizing the line, with a nominal voltage and no load. In the simulation the parameters obtained in the conventional tests, the distributed parameter model of the transmission line and the transient voltage event are used. Finally, the MATLAB-Simulink Parameter Estimation optimization tool is used to tune the transmission line parameters, so the signal of the simulation resembles as close as possible to the experimental response.
Resumen

En este artículo se presenta la estimación de los parámetros eléctricos de una línea de transmisión trifásica a partir de mediciones de transitorios de voltaje en un modelo de laboratorio a escala utilizando métodos de optimización no lineal. El objetivo del estudio busca obtener los parámetros de forma más precisa y rápida en comparación con los métodos convencionales. Las pruebas convencionales de circuito abierto y corto circuito se realizaron en un modelo a escala en el Laboratorio de Máquinas Eléctricas de la Escuela Politécnica Nacional y se utilizaron para obtener los parámetros eléctricos iniciales del modelo. Posteriormente, se simula un evento de voltaje transitorio mediante la energización de la línea utilizando un interruptor que proporciona una señal de voltaje experimental. La señal es capturada por un instrumento de medición para su posterior análisis. Con la ayuda de MATLAB-Simulink, la simulación de la línea de transmisión se realiza con el respectivo transitorio de voltaje obtenido al energizar la línea, con un voltaje nominal y sin carga. En la simulación se utilizan los parámetros obtenidos de manera convencional, el modelo de parámetros distribuidos de la línea de transmisión y el evento de voltaje transitorio. Finalmente, se utiliza la herramienta de optimización Parameter Estimation de MATLABSimulink para sintonizar la señal de la respuesta experimental y la simulación.

Palabras clave- Línea de transmisión de alto voltaje, sintonización de parámetros, sobrevoltajes transitorios, optimización no lineal.
Index terms- High voltage transmission line, parameter estimation, transient voltage, nonlinear optimization. 


\section{INTRODUCCIÓN}

En la solución de los problemas asociados a los estudios con líneas de transmisión se asume que los parámetros de la línea se conocen. La determinación más acertada de sus parámetros depende del objetivo del análisis que se realizará en el sistema eléctrico de potencia, el modelo de una línea de transmisión no será el mismo para un estudio del sistema en estado estable que para un estudio de coordinación de aislamiento y el conocimiento de las condiciones transitorias y posibles sobrevoltajes en la línea [1], [2], [3].

Los parámetros eléctricos de una línea de transmisión pueden ser calculados a partir de su geometría y sus componentes, pero la determinación de este modelo basado en los métodos de las funciones de Bessel y Carson producen ciertas imprecisiones debido a las aproximaciones que se utilizan para las condiciones ambientales variantes, la conductividad no homogénea del suelo y la geometría de la torre [2], [4].

Los parámetros de la línea pueden estimarse a partir de mediciones de voltaje y corriente en sus terminales. Quizá uno de los limitantes en la práctica es el instrumento que permita obtener esta medición, se puede utilizar unidades de medición fasorial PMUs o registros de fallas obtenidos de los relés de protecciones de la línea [1], [5]. Si bien es cierto existen diversos métodos para la estimación de parámetros, se debe tener en cuenta que la efectividad de los mismos difiere dependiendo de las características del sistema eléctrico, la geometría de la línea y la dinámica del sistema, de todas formas, cada método de estimación es efectivo para un punto de operación específico del sistema eléctrico y típicamente son pocos los parámetros de las matrices de admitancia e impedancia que se pueden estimar con precisión [6], [7].

Cuando el modelo de la línea se va a utilizar para realizar estudios eléctricos en estado transitorio, se ha reconocido ampliamente que los modelos clásicos de parámetros concentrados constantes no producen resultados satisfactorios pues tienen una independencia de la frecuencia que en el estado transitorio puede tomar un rango amplio de valores. Para afrontar este aspecto se han desarrollado con el paso de los años modelos dependientes de la frecuencia que permiten obtener de mejor manera los resultados de las simulaciones en estado transitorio conocidos como modelos de parámetros distribuidos que son de utilidad para el análisis de fallas o la coordinación del aislamiento en el sistema eléctrico [8].

Teniendo en cuenta los aspectos mencionados, este estudio se enfoca en obtener el modelo de parámetros distribuidos de una línea de transmisión a partir de la medición de un sobrevoltaje transitorio de maniobra. La ejecución del experimento en el sistema eléctrico conlleva muchas limitaciones y riesgos debido a los sobrevoltajes generados y la desconexión y reconexión de las líneas de transmisión, y por ello, se recurrió a un modelo de línea de transmisión a escala que funciona con $220 \mathrm{~V}$ en un laboratorio. De manera que en el modelo se pueden ejecutar varias veces el experimento para producir el sobrevoltaje necesario y poder determinar los parámetros de la línea en estudio.

De esta manera, este artículo técnico provee un ejemplo de la obtención de los parámetros de una línea de transmisión para que pueda ser empleado como un método alternativo para obtener los parámetros de una línea real en el sistema eléctrico cuando se obtenga un registro de un sobrevoltaje de maniobra, de manera que se mejore el modelo de aquella línea y pueda ser más acertado para los estudios en estado transitorio del sistema eléctrico al que pertenezca.

Este artículo contiene la parte práctica en la que se consiguieron los valores de entrada y salida utilizando un prototipo de laboratorio de línea de transmisión de $230 \mathrm{kV}$ que tiene longitudes de línea de $100 \mathrm{~km}, 200$ km y $400 \mathrm{~km}$; sus valores fueron comparados con los valores teóricos y después de un estudio minucioso se sintonizan los modelos mediante algoritmos de optimización de la Herramienta Parameter Estimation de MATLAB.

\section{PARÁMETROS DE LA LÍNEA DE TRANSMISIÓN}

Para la facilidad en los cálculos de los parámetros eléctricos de cualquier línea de transmisión se efectúan simplificaciones que van a depender de la longitud de la línea; por tal motivo las líneas se clasifican en: línea corta con una longitud de hasta $80 \mathrm{~km}$, línea media con una longitud comprendida entre $80 \mathrm{~km}$ y $240 \mathrm{~km}$ y línea larga con una longitud superior a $240 \mathrm{~km}$ [9].

Las líneas de transmisión están conformadas esencialmente por cuatro parámetros principales, los que están conectados tanto en serie como en paralelo, los parámetros que se encuentran conectados en serie son la resistencia y la inductancia formando una impedancia en serie al momento de dividirse de manera uniforme a lo largo de la línea; y los parámetros que se encuentran conectados en paralelo son la capacitancia y la conductancia. Cabe hacer mención que en las líneas de disposición aérea la conductancia en gran parte de los casos es despreciada [10], [11].

La línea de transmisión con sus respectivos parámetros eléctricos queda modelada como se representa en la Fig.1.

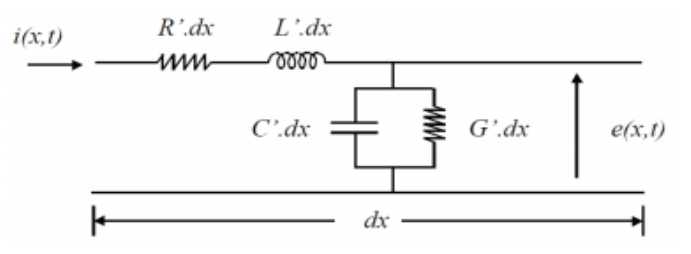

Figura 1: Infinitesimal de línea de transmisión [12] 
Siendo:

$\mathrm{R}^{\prime}$ : Resistencia en $\Omega / \mathrm{km}$.

L': Inductancia en $\mathrm{H} / \mathrm{km}$.

$\mathrm{C}^{\prime}$ : Capacitancia en $\mathrm{F} / \mathrm{km}$.

$\mathrm{G}^{\prime}$ : Conductancia en $\mu \mathrm{S} / \mathrm{km}$.

\subsection{Modelos de la línea de transmisión}

Dentro de los diferentes modelos existentes en la bibliografía se pueden encontrar los modelos de parámetros concentrados constantes como el modelo PI nominal y los modelos de parámetros distribuidos como el de Bergeron, estos modelos fueron los utilizados en este artículo [3].

\subsubsection{Modelo PI nominal}

Este modelo es el que está destinado a utilizarse en simulaciones de estado estacionario de las líneas cortas a una frecuencia nominal o frecuencia de la red. El circuito PI nominal para la línea de transmisión se representa en la Fig. 2. Las ecuaciones que hacen relación a las corrientes y voltajes en cada extremo de la línea se presentan en [3].

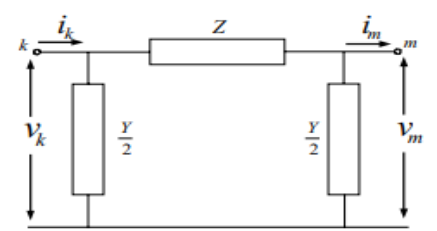

Figura 2: Circuito equivalente del modelo PI nominal de un vano de la línea de transmisión [3]

1 = Longitud de la línea en $\mathrm{km}$.

$\mathrm{Z}=$ Impedancia de la línea de transmisión.

Y= Admitancia de la línea de transmisión.

Este modelo presentado no suele tener tanta veracidad para fenómenos determinados por sobrevoltajes por maniobra y por descargas atmosféricas [3].

\subsubsection{Modelo de Bergeron}

Es un modelo elaborado por Hermann Dommel, se fundamenta en fenómenos de difusión de ondas viajeras presentes en la línea de transmisión, usando parámetros constantes y distribuidos a lo largo de la línea L' y C'. En la Fig. 3 se presenta el modelo mencionado y las ecuaciones que hacen relación a las corrientes y voltajes en cada extremo de la línea se presentan en [3].

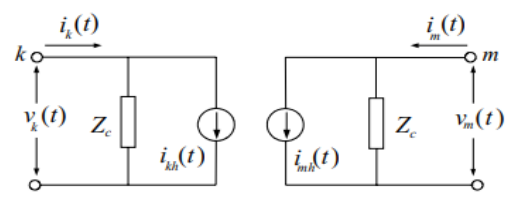

Figura 3: Modelo de Bergeron para una línea de transmisión [3]

\subsection{Métodos de optimización no lineales en MATLAB-Simulink usados para optimizar}

En la actualidad existen muchos tipos de métodos que proporcionan la optimización de funciones establecidas mediante un ajuste de curvas. En este artículo para la optimización de las respectivas funciones se tomó como base la utilización de los métodos que facilita el software MATLAB-Simulink en su herramienta Parameter Estimation, los mismos que se pueden referir con mayor detalle en [13].

\section{METODOLOGÍA}

Para realizar la estimación de los parámetros del modelo a escala de línea de transmisión se procedió según el diagrama de flujo de la Fig. 4.

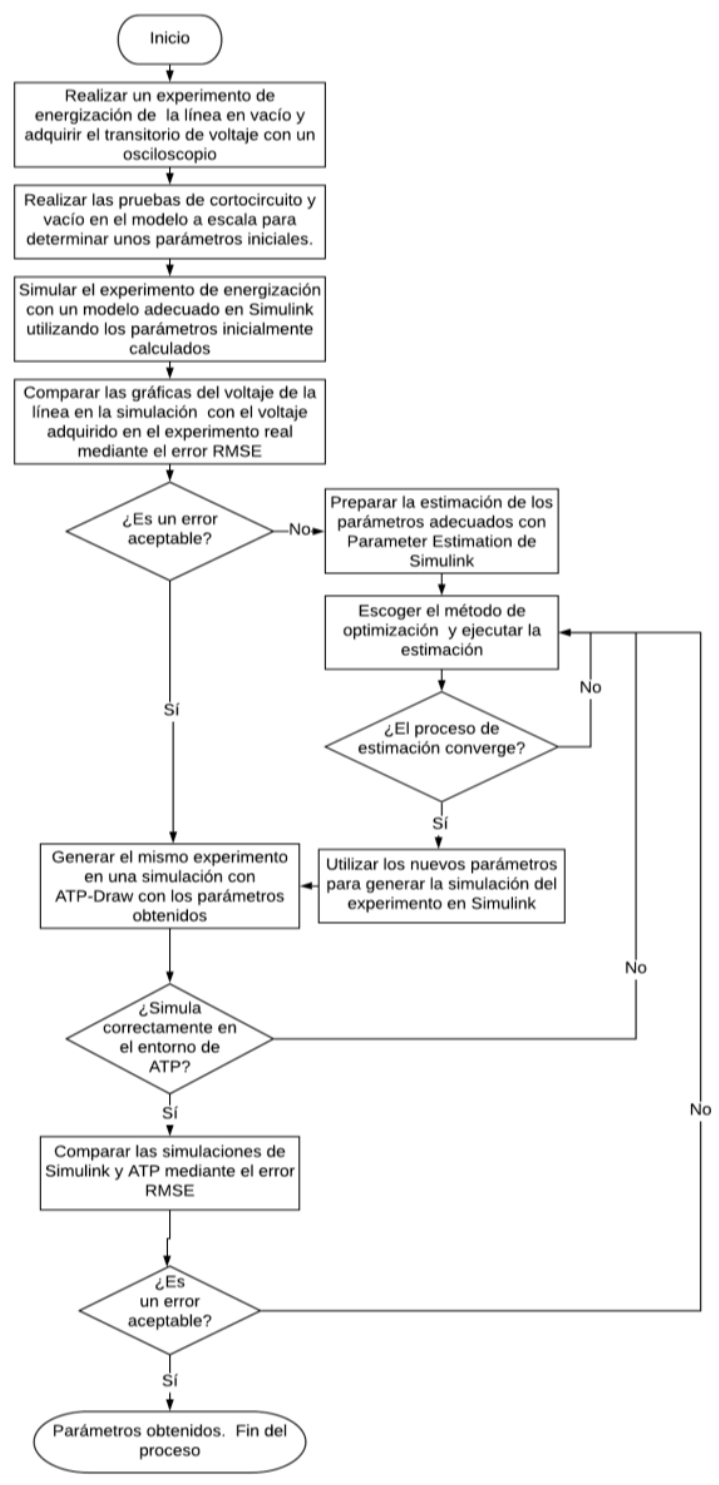

Figura 4: Procedimiento de estimación de los parámetros de la línea de transmisión

Los componentes necesarios para este procedimiento se describen a continuación. 


\subsection{Modelo de simulación}

Se utilizó el modelo de parámetros distribuidos existente en la biblioteca de MATLAB-Simulink, porque ha sido comprobado con validez tanto teórica como práctica, además se adecúa al modelos a escala de línea de transmisión trifásica que se tiene en el laboratorio y consigue tratar la mayoría de parámetros eléctricos encontrados en las pruebas de laboratorio, el modelo denominado como "Distributed Parameters Line", que considera a la línea trifásica con parámetros distribuidos [14].

\subsection{Modelo a escala de laboratorio}

Se utilizó un prototipo de línea de transmisión trifásica de $230 \mathrm{kV}$ con la que se puede trabajar con diferentes longitudes para el análisis; específicamente se usó para 100 km, 200 km y 400 km y está disponible en el Laboratorio de Máquinas Eléctricas de la Escuela Politécnica Nacional.

Se efectuaron pruebas de corto circuito y circuito abierto en donde se tomaron datos de voltajes de envío y de recepción, además de corrientes de envío y de recepción para cada longitud de la línea. Esta información era necesaria para poder obtener los valores de los parámetros eléctricos de la línea de transmisión y compararlos con los parámetros que corresponden a una línea de transmisión de $200 \mathrm{~km}$, que se encuentran en el documento en el que se detalla la construcción de dicha línea en [15].

Para las líneas de transmisión de 100 km y 200 km se utilizan las ecuaciones (1), (2), (3), (4), (5), (6) y (7), siendo $V_{S}$, $I_{S}$ los voltajes y corrientes de envío y $V_{R}, I_{R}$ los voltajes y corrientes de recepción.

$$
\begin{aligned}
& {\left[\begin{array}{l}
V S \\
I S
\end{array}\right]=\left[\begin{array}{ll}
A & B \\
C & D
\end{array}\right]\left[\begin{array}{l}
V_{R} \\
I_{R}
\end{array}\right]} \\
& \boldsymbol{V} \boldsymbol{s}=\boldsymbol{A} \boldsymbol{V}_{\boldsymbol{R}}+\boldsymbol{B} \boldsymbol{I}_{\boldsymbol{R}} \\
& \boldsymbol{I} \boldsymbol{s}=\boldsymbol{C V}_{\boldsymbol{R}}+\boldsymbol{D} \boldsymbol{I}_{\boldsymbol{R}} \\
& \boldsymbol{A}=\frac{Z \boldsymbol{Y}}{2}+\mathbf{1} \\
& \boldsymbol{B}=\boldsymbol{Z} \\
& \boldsymbol{C}=\boldsymbol{Y}\left(\frac{Z \boldsymbol{Y}}{\mathbf{4}}+\mathbf{1}\right) \\
& \boldsymbol{D}=\frac{Z Y}{2}+\mathbf{1}
\end{aligned}
$$

Para la línea de transmisión de 400 km se utilizan las ecuaciones (1), (2), (3), (8), (9), (10) y (11).

$$
\begin{gathered}
A=\frac{Z^{\prime} Y^{\prime}}{2}+1 \\
B=Z^{\prime} \\
C=Y^{\prime}\left(\frac{Z^{\prime} Y^{\prime}}{4}+1\right) \\
D=\frac{Z^{\prime} Y^{\prime}}{2}+1
\end{gathered}
$$

Para obtener los parámetros de secuencia cero se hace uso de las ecuaciones (12), (13), (14), (15), (16), (17) y (18) según [16].

$$
\begin{aligned}
& \boldsymbol{Z}_{\mathbf{0 1 2}}=\left[\begin{array}{ccc}
Z+2 M & & \\
& Z-M & \\
Z_{0}=Z_{S}+2 Z_{M} & Z-M
\end{array}\right] \\
& Z_{0}=\left(R_{P}+3 R_{G}\right)+j\left(X_{S}+2 X_{M}+3 X_{G}\right) \\
& Z_{1}=Z_{S}-Z_{M} \\
& Z_{1}=R_{P}+j\left(X_{S}-X_{M}\right) \\
& Z_{S}=\frac{Z_{0}+2 Z_{1}}{3} \\
& Z_{M}=\frac{Z_{0}-Z_{1}}{3}
\end{aligned}
$$

\subsection{Generación del evento transitorio de voltaje}

El evento transitorio de voltaje que se efectúa es el de energización en vacío de la línea de transmisión trifásica, para lo cual se hace uso de un interruptor trifásico. Este elemento es conectado entre la fuente de alimentación y la línea de transmisión y para efectuar el evento se cierra de manera manual energizando el circuito y produciendo en ese instante de energización el transitorio de voltaje. El circuito implementado se presenta en la Fig. 5.

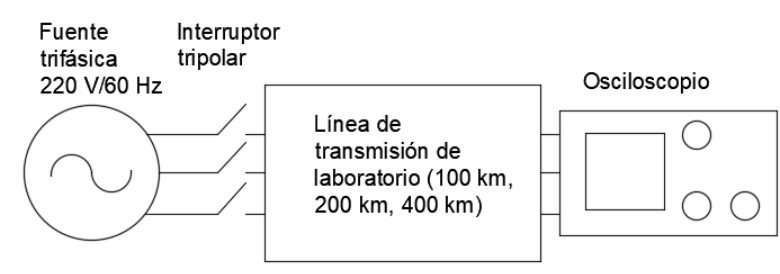

Figura 5: Circuito implementado en el Laboratorio de Máquina Eléctricas de la EPN

Se escogió una maniobra de energización de una línea por la sencillez en su ejecución. La forma de la onda de sobrevoltaje depende del instante de tiempo en el que se cierra el interruptor que energiza la línea; la maniobra no posee un control de tiempo de cierre del interruptor ya que es manual, y en general mientras más cercano sea el tiempo de cierre del interruptor al tiempo de cruce por cero de la onda de voltaje de la fuente se produce un sobrevoltaje menor en la línea de transmisión. Observando una serie de experimentos se consideró que mientras más cercano sea el tiempo de cierre al tiempo de un pico de la onda de voltaje de la fuente el sobrevoltaje en la línea era mayor y más evidente, y en la simulación que se realizaría posteriormente se podía modificar fácilmente el tiempo del cierre del interruptor hasta que el sobrevoltaje simulado tenga la misma fase que el sobrevoltaje medido en el experimento, al ser más pronunciado el transitorio de voltaje se podría estimar de mejor manera los valores de los parámetros del modelo de la línea que tienen mayor influencia en la forma de la onda, por ello se proceden a realizar varios de estos eventos hasta conseguir un transitorio pronunciado que tenga una forma de onda que sea evidente para poder realizar el trabajo propuesto. Las maniobras de energización se las realiza para las tres longitudes de línea que son: $100 \mathrm{~km}$, $200 \mathrm{~km}$ y $400 \mathrm{~km}$. 
3.4. La adquisición de datos se realizó mediante la obtención de las formas de onda de las curvas de entrada y curvas de salida usando un osciloscopio digital por medio de dos de sus canales, estos datos se recopilaron digitalmente para su posterior utilización.

\subsection{Estimación de parámetros}

Las pruebas efectuadas de circuito abierto y de corto circuito correspondientes, sirvieron para calcular los parámetros eléctricos iniciales de las líneas de transmisión según lo indicado en la sección 3.2 y asî conseguir las señales de las curvas de voltaje a las salidas de cada línea con la asistencia del osciloscopio digital.

Los datos fueron procesados usando MATLABSimulink, y se modeló la línea de transmisión con su respectiva perturbación, el modelo está conformado por una fuente de voltaje trifásico alterno que proporciona el voltaje adecuado, la línea de transmisión trifásica de parámetros distribuidos, un interruptor que servirá para realizar los eventos transitorios de voltaje por maniobra en el sistema, y un medidor de voltaje de salida que indicará la cantidad de voltaje que se obtiene y la forma de la señal.

Una vez inicializados los parámetros eléctricos de la línea de transmisión se procede con la simulación y se obtienen como resultado dos curvas; una que representa a la curva obtenida mediante la simulación con las condiciones iniciales, la misma que se utilizará en una optimización para la estimación de los parámetros eléctricos y la otra que es la curva obtenida de forma experimental en el laboratorio, mediante las mediciones descritas en 3.3.

La sintonización de parámetros se efectúa con la ayuda de la herramienta de Estimación de Parámetros de Simulink. Para detalles de cómo usar esta herramienta de estimación y los diferentes métodos de optimización no lineales que existen referirse a [17].

\section{RESULTADOS Y DISCUSIÓN}

4.1. Cálculo de los parámetros eléctricos de secuencia positiva en base a las pruebas de circuito abierto y de corto circuito

En este caso se dispone de dos líneas medias de longitudes correspondientes a $100 \mathrm{~km}$ y $200 \mathrm{~km}$; y una línea larga de $400 \mathrm{~km}$. Los resultados de las pruebas de vacío efectuadas se presentan en la Tabla 1 y de las pruebas de cortocircuito, en la Tabla 2. Para obtener los parámetros de la línea se procede según la Sección 3.2 y se obtienen los resultados iniciales presentados en la Tabla 3.

Se ejecuta la simulación del evento transitorio de voltaje y la Fig. 6 representa los resultados iniciales de las curvas experimental (roja) y simulada (azul) en el caso de la línea de $400 \mathrm{~km}$ para poder aplicar a estas señales los diferentes métodos de optimización mediante la herramienta Parameter Estimation.

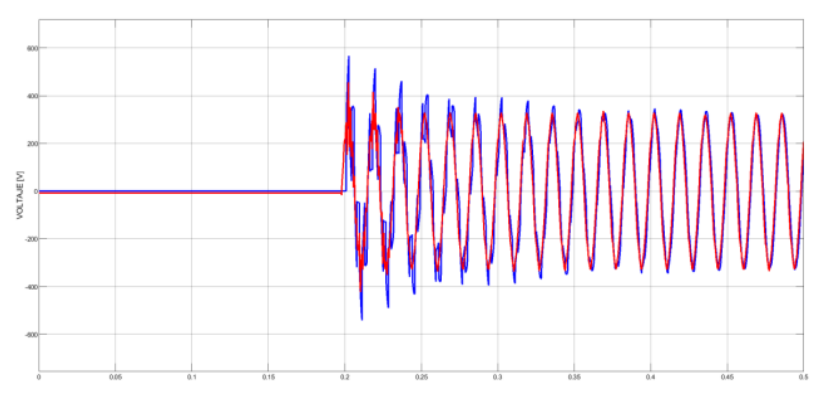

Figura 6: Voltaje en la línea de transmisión trifásica de 400 km de forma experimental (roja) vs Simulación (azul)

4.2. Sintonización de los parámetros eléctricos de la línea de transmisión trifásica mediante el evento transitorio de voltaje

En la herramienta Parameter Estimation de MATLAB se realizan las sintonizaciones de los parámetros con los diversos métodos de optimización que posee que son: Levenberg-Marquardt, Región De Confianza Reflectivo, Búsqueda Simple, Programación Cuadrática Secuencial y Conjunto Activo. En la Fig. 7 se observa como ejemplo el ajuste realizado por el método Levenberg-Marquardt para que la señal simulada sea similar a la señal experimental para el caso de la línea de $400 \mathrm{~km}$.

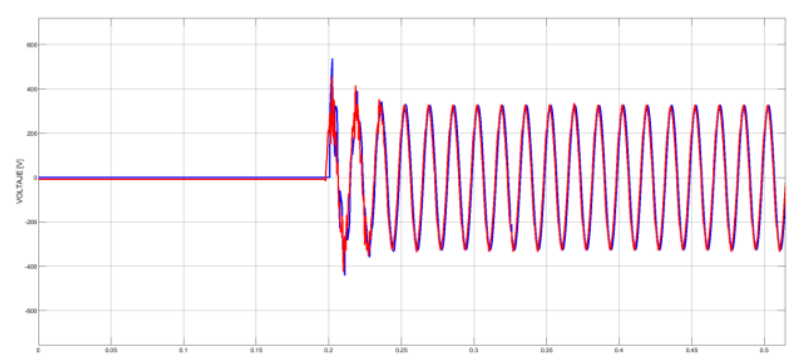

Figura 7: Voltaje en la línea de transmisión trifásica de 400 km después de la estimación de parámetros por el método LevenbergMarquardt, Experimental (roja) vs Simulación (azul)

En la Tabla 4 se encuentran los resultados de parámetros sintonizados al aplicar los diferentes métodos de optimización. Los resultados del método Levenberg Marquardt indican que existe en general una variación muy pequeña en gran parte de los parámetros eléctricos de secuencia positiva y cero lo que produce un porcentaje de diferencia muy pequeña, siendo la excepción la resistencia de secuencia positiva la cual posee un porcentaje de diferencia grande.

En los resultados obtenidos al aplicar el método Región de Confianza Reflectivo se evidencia que existe una variación de los parámetros eléctricos de secuencia positiva con un porcentaje de diferencia pequeño, siendo la más considerable de la resistencia de secuencia positiva, para los parámetros eléctricos de 
Tabla 1: Resultados de las pruebas de circuito abierto en la línea de transmisión

\begin{tabular}{|c|c|c|c|c|c|c|c|c|c|}
\hline \multicolumn{9}{|c|}{ Prueba de Circuito Abierto } \\
\hline & \multicolumn{3}{c|}{$\begin{array}{c}\text { Voltaje de } \\
\text { envío en V }\end{array}$} & \multicolumn{3}{c|}{$\begin{array}{c}\text { Corriente de } \\
\text { línea en A }\end{array}$} & \multicolumn{3}{c|}{$\begin{array}{c}\text { Voltaje de } \\
\text { recepción en V }\end{array}$} \\
\hline $\begin{array}{c}\text { Longitud } \\
\text { en km }\end{array}$ & VAB & VBC & VCA & IA & IB & IC & VAB & VBC & VCA \\
\hline $\mathbf{1 0 0}$ & 208 & 211 & 211 & 1,2 & 1,3 & 1,4 & 210 & 214 & 213 \\
\hline $\mathbf{2 0 0}$ & 208,7 & 212,7 & 212,3 & 3 & 3,1 & 3,2 & 217,4 & 222,2 & 221,5 \\
\hline $\mathbf{4 0 0}$ & 210,3 & 212,6 & 209,2 & 6,5 & 6,6 & 6,7 & 248 & 248 & 246 \\
\hline
\end{tabular}

Tabla 2: Resultados de las pruebas de corto circuito en la línea de transmisión

\begin{tabular}{|c|c|c|c|c|c|c|c|c|c|c|c|c|}
\hline \multicolumn{13}{|c|}{ Prueba de Corto Circuito } \\
\hline & \multicolumn{3}{|c|}{$\begin{array}{l}\text { Corriente de } \\
\text { envío en A }\end{array}$} & \multicolumn{3}{|c|}{$\begin{array}{l}\text { Corriente de } \\
\text { recepción en A }\end{array}$} & \multicolumn{3}{|c|}{$\begin{array}{c}\text { Resistencia por } \\
\text { fase medidas en } \mathrm{m} \Omega\end{array}$} & \multicolumn{3}{|c|}{$\begin{array}{c}\text { Voltaje de } \\
\text { entrada en } V\end{array}$} \\
\hline $\begin{array}{l}\text { Longitud } \\
\text { en km }\end{array}$ & IA & IB & IC & IA & IB & IC & $\mathbf{R A}$ & RB & $\mathbf{R C}$ & VAB & VBC & VCA \\
\hline 100 & 15 & 14,9 & 14,7 & 15,1 & 15 & 14,8 & 100 & 126,8 & 102,98 & 36,8 & 36,6 & 37,3 \\
\hline 200 & 14,8 & 14,5 & 14,7 & 15,2 & 15 & 15 & 202,8 & 267,7 & 233,4 & 74 & 74,4 & 74,3 \\
\hline 400 & 13,3 & 13,3 & 13,6 & 15,4 & 15,1 & 15,4 & 367,7 & 384,2 & 386,7 & 134 & 136,4 & 134,8 \\
\hline
\end{tabular}

secuencia cero no existe variación por lo que posee un porcentaje de diferencia nula en todos los casos.

Tabla 3: Tabla de parámetros iniciales para las líneas de transmisión de diferentes longitudes

\begin{tabular}{|l|c|c|c|c|c|c|}
\multicolumn{4}{c|}{} & \multicolumn{4}{c|}{ transmisión de diferentes longitudes } \\
\cline { 2 - 7 } \multicolumn{1}{c|}{} & \multicolumn{3}{|c|}{$\begin{array}{c}\text { Parámetros iniciales de las líneas de transmisión } \\
\text { trifásicas }\end{array}$} \\
\cline { 2 - 7 } & $\begin{array}{c}\text { Línea de } \\
\text { transmisión de } \\
\mathbf{1 0 0} \mathbf{~ k m}\end{array}$ & \multicolumn{2}{c|}{$\begin{array}{c}\text { Línea de } \\
\text { transmisión de } \\
\mathbf{2 0 0} \mathbf{~ k m}\end{array}$} & \multicolumn{2}{c|}{$\begin{array}{c}\text { Línea de } \\
\text { transmisión de } \\
\mathbf{4 0 0} \mathbf{~ k m}\end{array}$} \\
\cline { 2 - 7 } & Sec 0 & Sec. 1, 2 & Sec. 0 & Sec. 1, 2 & Sec. 0 & Sec. 1, 2 \\
\hline $\mathbf{L}$ & 11,2 & 3,7 & 20,45 & 6,84 & 38,51 & 12,83 \\
\hline$[\mathrm{mH}]$ & & & & & & \\
\hline $\mathbf{R}$ & 0,2 & 0,1 & 0,5 & 0,25 & 0,74 & 0,3 \\
{$[\Omega]$} & & & & & & \\
\hline $\mathbf{C}$ & 6,53 & 51,51 & 18,75 & 38 & 29,06 & 58 \\
\hline$\mu \mathrm{F}]$
\end{tabular}

El método de Búsqueda Simple expresa los resultados con un porcentaje de diferencia elevado tanto para los parámetros de secuencia positiva y cero, siendo la más llamativa para la inductancia de secuencia positiva ya que tiene un valor considerable de variación.

Para el algoritmo Programación Cuadrática Secuencial se tienen variaciones para los parámetros eléctricos de secuencia positiva siendo estas las de mayor porcentaje de diferencia, teniendo para la inductancia un valor muy elevado. En mención a los parámetros eléctricos de secuencia cero las variaciones son prácticamente nulas por lo cual no existe porcentaje de diferencia en estos casos.

El método Conjunto Activo presenta los resultados con un porcentaje de diferencia nulo, prácticamente en todos los parámetros eléctricos debido a que dicho método no ejecuta muchas iteraciones haciendo que la inductancia, resistencia y capacitancia tanto para secuencia positiva y cero no sufran gran variación.

\subsection{Selección del método más apropiado para la sintonización de parámetros eléctricos de líneas de transmisión trifásicas de diferentes longitudes según el error cuadrático medio}

En las pruebas realizadas para la obtención de los parámetros eléctricos originales de la línea de transmisión se presentan errores ya sea por apreciación humana o por los errores de los equipos de medición.

Para escoger el método de optimización no lineal adecuado, en primera instancia se procedió a tomar como base el error cuadrático medio entre las curvas simuladas y reales de voltaje de las líneas de transmisión propuestas. Luego, con la ayuda del software ATP/ATPDraw se puede corroborar si los parámetros seleccionados son los adecuados, ya que este programa proporciona mayor confiabilidad al momento de simular transitorios electromagnéticos, en este caso de la línea de transmisión con los parámetros estimados; teniendo así los siguientes resultados.

En la Tabla 5 se presenta el error cuadrático medio RMSE de la señal simulada. 
Tabla 4: Parámetros eléctricos estimados con diferentes métodos para la línea de transmisión trifásica

\begin{tabular}{|c|c|c|c|c|c|c|c|c|c|c|c|}
\hline \multicolumn{12}{|c|}{ LÍNEA DE TRANSMISIÓN DE $100 \mathrm{~km}$} \\
\hline \multirow{2}{*}{\multicolumn{2}{|c|}{$\begin{array}{l}\text { PARÁMETROS } \\
\text { CALCULADOS }\end{array}$}} & \multicolumn{2}{|c|}{$\begin{array}{l}\text { LEVENBERG- } \\
\text { MARQUARDT }\end{array}$} & \multicolumn{2}{|c|}{$\begin{array}{c}\text { REGIÓN DE } \\
\text { CONFIANZA } \\
\text { REFLECTIVO }\end{array}$} & \multicolumn{2}{|c|}{$\begin{array}{l}\text { BÚSQUEDA } \\
\text { SIMPLE }\end{array}$} & \multicolumn{2}{|c|}{$\begin{array}{l}\text { PROGRAMACIÓN } \\
\text { CUADRÁTICA } \\
\text { SECUENCIAL }\end{array}$} & \multicolumn{2}{|c|}{$\begin{array}{c}\text { CONJUNTO } \\
\text { ACTIVO }\end{array}$} \\
\hline & & \multirow{2}{*}{$\begin{array}{c}\text { Estimados } \\
5,45 \mathrm{mH} \\
\end{array}$} & \multirow{2}{*}{\begin{tabular}{c|c}
$\begin{array}{c}\text { Error } \\
\text { relativo } \\
\text { en \% }\end{array}$ \\
32,11 \\
\end{tabular}} & \multirow{2}{*}{$\begin{array}{c}\text { Estimados } \\
1,2 \mathrm{mH} \\
\end{array}$} & \multirow{2}{*}{$\begin{array}{c}\begin{array}{c}\text { Error } \\
\text { relativo } \\
\text { en \% }\end{array} \\
208,33 \\
\end{array}$} & \multirow{2}{*}{$\begin{array}{c}\text { Estimados } \\
0,0088 \mathrm{mH} \\
\end{array}$} & \multirow{2}{*}{$\begin{array}{c}\begin{array}{c}\text { Error } \\
\text { relativo } \\
\text { en \% }\end{array} \\
41945,45 \\
\end{array}$} & \multirow{2}{*}{$\begin{array}{c}\text { Estimados } \\
0,0095 \mathrm{mH} \\
\end{array}$} & \multirow{2}{*}{\begin{tabular}{|c|}
$\begin{array}{c}\text { Error } \\
\text { relativo } \\
\text { en \% }\end{array}$ \\
38847,37 \\
\end{tabular}} & \multirow{2}{*}{$\begin{array}{c}\text { Estimados } \\
3,7 \mathrm{mH} \\
\end{array}$} & \multirow{2}{*}{$\begin{array}{c}\begin{array}{c}\text { Error } \\
\text { relativo en } \\
\%\end{array} \\
0,00 \\
\end{array}$} \\
\hline $\mathbf{L 1}$ & $3,7 \mathrm{mH}$ & & & & & & & & & & \\
\hline LO & $11,2 \mathrm{mH}$ & $11,14 \mathrm{mH}$ & 0,54 & $11,2 \mathrm{mH}$ & 0,00 & $7,02 \mathrm{mH}$ & 59,54 & $11,2 \mathrm{mH}$ & 0,00 & $11,2 \mathrm{mH}$ & 0,00 \\
\hline R1 & $0,1 \Omega$ & $14,52 \Omega$ & 99,3 & $3,23 \Omega$ & 96,90 & $0,17 \Omega$ & 41,18 & $0,25 \Omega$ & 60,00 & $0,1 \Omega$ & 0,00 \\
\hline RO & $0,2 \Omega$ & $0,2 \Omega$ & 0,0 & $0,2 \Omega$ & 0,00 & $0,23 \Omega$ & 13,04 & $0,2 \Omega$ & 0,00 & $0,21 \Omega$ & 4,76 \\
\hline C1 & $13 \mu \mathrm{F}$ & $51,51 \mu \mathrm{F}$ & 74,8 & $231,37 \mu \mathrm{F}$ & 94,38 & $30 \mu \mathrm{F}$ & 56,67 & $17,5 \mu \mathrm{F}$ & 25,71 & $13 \mu \mathrm{F}$ & 0,00 \\
\hline CO & $6,53 \mu \mathrm{F}$ & $6,42 \mu \mathrm{F}$ & 1,71 & $6,53 \mu \mathrm{F}$ & 0,00 & $3,97 \mu \mathrm{F}$ & 64,48 & $6,53 \mu \mathrm{F}$ & 0,00 & $6,53 \mu \mathrm{F}$ & 0,00 \\
\hline \multicolumn{12}{|c|}{ LÍNEA DE TRANSMISIÓN DE $200 \mathrm{~km}$} \\
\hline \multirow{2}{*}{\multicolumn{2}{|c|}{$\begin{array}{l}\text { PARÁMETROS } \\
\text { CALCULADOS }\end{array}$}} & \multicolumn{2}{|c|}{$\begin{array}{l}\text { LEVENBERG- } \\
\text { MARQUARDT }\end{array}$} & \multicolumn{2}{|c|}{$\begin{array}{c}\text { REGIÓN DE } \\
\text { CONFIANZA } \\
\text { REFLECTIVO } \\
\end{array}$} & \multicolumn{2}{|c|}{$\begin{array}{l}\text { BÚSQUEDA } \\
\text { SIMPLE }\end{array}$} & \multicolumn{2}{|c|}{$\begin{array}{l}\text { PROGRAMACIÓN } \\
\text { CUADRÁTICA } \\
\text { SECUENCIAL }\end{array}$} & \multicolumn{2}{|c|}{$\begin{array}{c}\text { CONJUNTO } \\
\text { ACTIVO }\end{array}$} \\
\hline & & Estimados & $\begin{array}{c}\text { Error } \\
\text { relativo } \\
\text { en \% }\end{array}$ & Estimados & $\begin{array}{l}\text { Error } \\
\text { relativo } \\
\text { en \% }\end{array}$ & Estimados & $\begin{array}{c}\text { Error } \\
\text { relativo } \\
\text { en \% }\end{array}$ & Estimados & $\begin{array}{c}\text { Error } \\
\text { relativo } \\
\text { en \% }\end{array}$ & Estimados & $\begin{array}{l}\text { Error } \\
\text { relativo } \\
\text { en \% }\end{array}$ \\
\hline $\mathbf{L 1}$ & $6,84 \mathrm{mH}$ & $7,91 \mathrm{mH}$ & 13,53 & $7,8 \mathrm{mH}$ & $12,31 \%$ & $2,57 \mathrm{mH}$ & 166,15 & $0,023 \mathrm{mH}$ & 29639,13 & $6,84 \mathrm{mH}$ & 0,00 \\
\hline LO & $20,45 \mathrm{mH}$ & $25,68 \mathrm{mH}$ & 20,37 & $20,44 \mathrm{mH}$ & $0,05 \%$ & $19,63 \mathrm{mH}$ & 4,18 & $40,45 \mathrm{mH}$ & 49,44 & $20,45 \mathrm{mH}$ & 0,00 \\
\hline R1 & $0,25 \Omega$ & $1,98 \Omega$ & 87,37 & $1,97 \Omega$ & $87,31 \%$ & $0,44 \Omega$ & 43,18 & $0,4 \Omega$ & 37,50 & $0,25 \Omega$ & 0,00 \\
\hline RO & $0,5 \Omega$ & $0,58 \Omega$ & 13,79 & $0,5 \Omega$ & $0,00 \%$ & $0,00097 \Omega$ & 51446,39 & $0,5 \Omega$ & 0,00 & $0,5 \Omega$ & 0,00 \\
\hline C1 & $38 \mu \mathrm{F}$ & $32,43 \mu \mathrm{F}$ & 17,18 & $32,86 \mu \mathrm{F}$ & $15,64 \%$ & $67,46 \mu \mathrm{F}$ & 43,67 & $40,08 \mu \mathrm{F}$ & 5,19 & $38 \mu \mathrm{F}$ & 0,00 \\
\hline $\mathrm{CO}$ & $18,75 \mu \mathrm{F}$ & $26,01 \mu \mathrm{F}$ & 27,91 & $18,75 \mu \mathrm{F}$ & $0,00 \%$ & $13,71 \mu \mathrm{F}$ & 36,76 & $18,75 \mu \mathrm{F}$ & 0,00 & $18,75 \mu \mathrm{F}$ & 0,00 \\
\hline \multicolumn{12}{|c|}{ LÍNEA DE TRANSMISIÓN DE $400 \mathrm{~km}$} \\
\hline \multirow{2}{*}{\multicolumn{2}{|c|}{$\begin{array}{l}\text { PARÁMETROS } \\
\text { CALCULADOS }\end{array}$}} & \multicolumn{2}{|c|}{$\begin{array}{l}\text { LEVENBERG- } \\
\text { MARQUARDT }\end{array}$} & \multicolumn{2}{|c|}{$\begin{array}{c}\text { REGIÓN DE } \\
\text { CONFIANZA } \\
\text { REFLECTIVO } \\
\end{array}$} & \multicolumn{2}{|c|}{$\begin{array}{l}\text { BÚSQUEDA } \\
\text { SIMPLE }\end{array}$} & \multicolumn{2}{|c|}{$\begin{array}{l}\text { PROGRAMACIÓN } \\
\text { CUADRÁTICA } \\
\text { SECUENCIAL } \\
\end{array}$} & $\begin{array}{r}\text { CONJU } \\
\text { ACTI }\end{array}$ & $\begin{array}{l}\text { UNTO } \\
\text { IVO }\end{array}$ \\
\hline & & Estimados & $\begin{array}{l}\text { Error } \\
\text { relativo } \\
\text { en \% }\end{array}$ & Estimados & $\begin{array}{c}\text { Error } \\
\text { relativo } \\
\text { en \% } \\
\end{array}$ & Estimados & $\begin{array}{l}\text { Error } \\
\text { relativo } \\
\text { en \% }\end{array}$ & Estimados & $\begin{array}{l}\text { Error } \\
\text { relativo } \\
\text { en \% }\end{array}$ & Estimados & $\begin{array}{l}\text { Error } \\
\text { relativo } \\
\text { en \% }\end{array}$ \\
\hline $\mathbf{L 1}$ & $12,83 \mathrm{mH}$ & $13,32 \mathrm{mH}$ & 3,68 & $13,26 \mathrm{mH}$ & 3,24 & $5,68 \mathrm{mH}$ & 125,88 & $0,0094 \mathrm{mH}$ & $\begin{array}{c}136389,3 \\
6\end{array}$ & $12,83 \mathrm{mH}$ & 0,00 \\
\hline LO & $38,51 \mathrm{mH}$ & $39,09 \mathrm{mH}$ & 1,48 & $38,51 \mathrm{mH}$ & 0,00 & $39,46 \mathrm{mH}$ & 2,41 & $38,51 \mathrm{mH}$ & 0,00 & $38,5 \mathrm{mH}$ & 0,03 \\
\hline $\mathbf{R} 1$ & $0,3 \Omega$ & $1,88 \Omega$ & 84,04 & $1,87 \Omega$ & 83,96 & $0,43 \Omega$ & 30,23 & $0,34 \Omega$ & 11,76 & $0,3 \Omega$ & 0,00 \\
\hline RO & $0,74 \Omega$ & $0,74 \Omega$ & 0,00 & $0,74 \Omega$ & 0,00 & $0,85 \Omega$ & 12,94 & $0,74 \Omega$ & 0,00 & $0,78 \Omega$ & 5,13 \\
\hline C1 & $58 \mu \mathrm{F}$ & $54,99 \mu \mathrm{F}$ & 5,47 & $55,04 \mu \mathrm{F}$ & 5,38 & $74,79 \mu \mathrm{F}$ & 22,45 & $35,81 \mu \mathrm{F}$ & 61,97 & $58 \mu \mathrm{F}$ & 0,00 \\
\hline $\mathrm{CO}$ & $29,06 \mu \mathrm{F}$ & $28,56 \mu \mathrm{F}$ & 1,75 & $29,06 \mu \mathrm{F}$ & 0,00 & $33,96 \mu \mathrm{F}$ & 14,43 & $29,06 \mu \mathrm{F}$ & 0,00 & $29,06 \mu \mathrm{F}$ & 0,00 \\
\hline
\end{tabular}

Tomando como ejemplo la línea de $400 \mathrm{~km}$, se observa que con los parámetros calculados inicialmente el valor RMSE es de 65,2. Una vez calculado este error en cada uno los métodos aplicados para la estimación de parámetros se pueden evidenciar que existe un algoritmo que logra reducirlo a un valor mínimo de 43,13 siendo este el de Programación Cuadrática Secuencial. Sin embargo, al utilizar los parámetros obtenidos por el método de Programación Cuadrática Secuencial y simularlos en el modelo de Bergeron de
ATP la simulación no pudo ser ejecutada mientras que en Simulink la simulación funcionaba correctamente.

En la Tabla 4 se puede apreciar que el método de Programación Cuadrática Secuencial difiere en gran medida en el valor de la inductancia de secuencia positiva $\mathrm{L}_{1}$, el método obtiene un valor de $\mathrm{L}_{1}$ muy pequeño comparado con los otros métodos y al usar este valor en ATP se producen errores debido a que el valor del parámetro es irreal. Se puede apreciar entonces que a pesar de que Simulink realice esta simulación con ese 
valor no necesariamente es una simulación correcta, también se aprecia que el método con el menor RMSE no necesariamente es el mejor método para obtener los parámetros de esta línea. Se puede decir que para la sintonización de parámetros eléctricos de una línea de transmisión trifásica de $400 \mathrm{~km}$ no es recomendable aplicar el método Programación Cuadrática Secuencial. Y para comprobar que los parámetros obtenidos en Simulink pueden utilizarse se ponen a prueba en una simulación similar en el software ATP en todos los casos analizados.

Tabla 5: Error cuadrático medio con diferentes métodos para la línea de transmisión trifásica

\begin{tabular}{|c|c|c|c|c|}
\cline { 2 - 5 } \multicolumn{2}{c|}{} & \multicolumn{3}{c|}{ RMSE } \\
\hline \multicolumn{1}{|c|}{ Longitud de la línea [km] } & $\mathbf{1 0 0}$ & $\mathbf{2 0 0}$ & $\mathbf{4 0 0}$ \\
\hline \multirow{4}{*}{$\begin{array}{c}\text { Sin } \\
\text { optimización }\end{array}$} & $\begin{array}{c}\text { DATOS } \\
\text { INICIALES }\end{array}$ & 45,83 & 57,12 & 65,2 \\
\hline \multirow{5}{*}{$\begin{array}{c}\text { Métodos de } \\
\text { optimización }\end{array}$} & $\begin{array}{c}\text { LEVENBERG- } \\
\text { MARQUARDT }\end{array}$ & 14,91 & 48,09 & 51,8 \\
\cline { 2 - 5 } & $\begin{array}{c}\text { BÚSQUEDA } \\
\text { SIMPLE } \\
\text { CONFIÓN DE }\end{array}$ & 27,34 & 46,98 & 43,72 \\
\cline { 2 - 5 } & $\begin{array}{c}\text { REFECTIVO } \\
\text { PROGRAMACIÓN } \\
\text { CUADÁTICA } \\
\text { SECUENCIAL }\end{array}$ & 27,35 & 43,27 & 43,13 \\
\cline { 2 - 5 } & $\begin{array}{c}\text { CONJUNTO } \\
\text { ACTIVO }\end{array}$ & 45,83 & 57,12 & 65,2 \\
\hline
\end{tabular}

Las simulaciones realizadas en ATP de las líneas de transmisión trifásicas con parámetros eléctricos sintonizados a diferentes longitudes sirven para poder llegar a la conclusión que no es siempre aconsejable aplicar métodos de optimización no lineal con el criterio de selección del menor error cuadrático medio, ya que estos algoritmos proporcionan ciertos valores de parámetros eléctricos muy pequeños lo que significaría que físicamente no son muy reales. En efecto, para poder seleccionar el método de optimización no lineal adecuado para la sintonización de los parámetros eléctricos de las líneas de transmisión analizadas se debería escoger parámetros sintonizados que no estén muy lejos de los parámetros calculados y esto se logra identificar con la ayuda del error relativo obtenido entre los parámetros calculados y los parámetros estimados; evidenciando si estos son correctos o no con la respectiva simulación en el programa ATP. El procedimiento para las líneas de $100 \mathrm{~km}$ y $200 \mathrm{~km}$ es el mismo que se encuentra detallado para la línea de 400 $\mathrm{km}$. Se puede decir que, para la sintonización de parámetros eléctricos de una línea de las líneas de transmisión trifásica de 100, 200 y $400 \mathrm{~km}$ según la Tabla 4 donde se analiza y escoge el método de optimización con menor porcentaje de diferencia para la sintonización de parámetros eléctricos; se sugiere aplicar los algoritmos Levenberg Marquardt y Región de Confianza Reflectivo.

Las simulaciones efectuadas en ATP con los parámetros eléctricos ya sintonizados según los algoritmos Levenberg Marquardt y Región de Confianza Reflectivo ayudan a concluir que para la sintonización de parámetros eléctricos de las diferentes líneas de transmisión es muy recomendable aplicar los métodos señalados, ya que se ha verificado cada uno de estos con las simulaciones efectuadas en el ATP.

Haciendo referencia al método Conjunto Activo que evidentemente posee el menor porcentaje de diferencia no es considerado como muy aplicable para la sintonización de parámetros ya que este algoritmo realiza muy pocas iteraciones lo que produce que los parámetros no se ajusten del mismo modo como lo hacen los métodos ya expuestos que son el Levenberg Marquardt y Región de Confianza Reflectivo.

El procedimiento para las líneas de $100 \mathrm{~km}$ y 200 $\mathrm{km}$ es el mismo como se encuentra detallado para la línea de $400 \mathrm{~km}$. La comparación se realiza para evidenciar si las señales simuladas tanto en MATLAB como en ATP son parecidas o si a su vez tienen alguna variación. Se presenta un ejemplo para la línea de transmisión trifásica de 400 km en las Fig. 8 y Fig. 9.

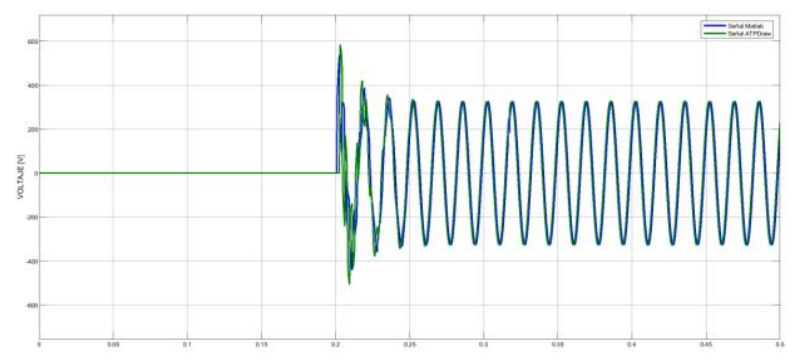

Figura 8: Voltaje en la línea de transmisión trifásica de 400 km después de la estimación de parámetros por el método Levenberg Marquardt, ATP (verde) vs MATLAB (azul)

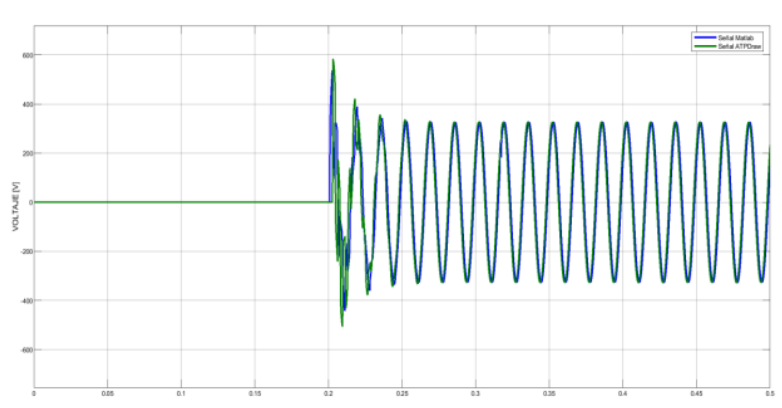

Figura 9: Voltaje en la línea de transmisión trifásica de 400 km después de la estimación de parámetros por el método Región de Confianza Reflectivo, ATP (verde) vs MATLAB (azul)

Finalmente; al realizar las simulaciones en los dos paquetes computacionales mencionados se puede 
observar que las señales obtenidas a las salidas de las líneas de transmisión trifásicas estudiadas son semejantes por lo que se puede aseverar que la sintonización de los parámetros eléctricos mediante la herramienta Parameter Estimation del Simulink es aplicable, especialmente con el uso de los métodos Levenberg Marquardt y Región de Confianza Reflectivo.

\section{CONCLUSIONES Y RECOMENDACIONES}

Para la simulación de la línea de transmisión se utilizó el modelo de parámetros distribuidos, ya que se lo consideró adecuado porque éste responde a perturbaciones como fenómenos transitorios producidos en la apertura y cierre de una línea de transmisión, dicha aseveración fue comprobada al efectuar las simulaciones en las cuales proporcionó resultados muy cercanos a la realidad. El modelo detallado es fundamental ya que ayudó a cumplir con uno de los objetivos del estudio que era estimar los parámetros de la línea ante la presencia de un transitorio de voltaje.

Al revisar los resultados obtenidos se puede decir que se cumple con el objetivo de estimar los parámetros de líneas de transmisión de una manera no tradicional, pudiendo tener una aplicación directa en las líneas de transmisión del Sistema Nacional de Transmisión en donde seria óptimo disponer de instrumentos que proporcionen información de las curvas de fenómenos transitorios, y así aprovechar estas señales y realizar la respectiva estimación de los parámetros.

El error cuadrático medio no siempre va a ser un indicador adecuado para poder seleccionar qué tipo de método de optimización no lineal es el mejor para realizar estimación de parámetros; ya que se pudo comprobar que el método de Programación Cuadrática Secuencial que tenía el menor error cuadrático medio en la línea de $400 \mathrm{~km}$ obtenía unos parámetros que no generan las simulaciones en otro programa que no sea Simulink como en el caso de ATP que se utiliza mucho para la simulación de transitorios de voltaje; ya que estos parámetros pueden ser muy pequeños por lo que se les considera inexistentes. Por ello en este trabajo se escogió los métodos que no generaban conflictos en las simulaciones con ATP junto a la observación de que su error RMSE y error relativo de parámetros sea aceptable.

\section{REFERENCIAS BIBLIOGRÁFICAS}

[1] C. S. Indulkar y K. Ramalingam, "Estimation of transmission line parameters from measurements", International Journal of Electrical Power \& Energy Systems, vol. 30, n.o 5, pp. 337-342, jun. 2008.

[2] E. C. M. Costa y S. Kurokawa, "Estimation of transmission line parameters using multiple methods", IET Generation Transmission \& Distribution. vol. 9, n.o 16, pp. 2617-2624, 2015.
[3] L. H. Restrepo, G. C. Delgado, y F. Castro Aranda, "Modelos de línea de transmisión para transitorios electromagnéticos en sistemas de potencia", Revista Energía y Computación, vol. 16, n.o 1, pp. 21-32, 2008.

[4] L. Hofmann, "Series expansions for line series impedances considering different specific resistances, magnetic permeabilities, and dielectric permittivities of conductors, air, and ground", IEEE Transactions on Power Delivery, vol. 18, n.o 2, pp. 564-570, abr. 2003.

[5] R. Zivanovic, "Estimation of transmission line parameters using fault records", Proceedings of the AUPEC 06, Melbourne, Australia, 2006.

[6] S. Kurokawa, G. A. Asti, E. C. M. Costa, y J. Pissolato, "Simplified procedure to estimate the resistance parameters of transmission lines", Electrical Engineering, vol. 95, n.o 3, pp. 221-227, sep. 2013.

[7] R. Schulze, P. Schegner, y P. Stachel, "Parameter identification of unsymmetrical transmission lines using accurately re-synchronised fault records", 2009 IEEE Power Tech, Bucharest,2009.

[8] J. R. Marti, "Accurate Modelling of FrequencyDependent Transmission Lines in Electromagnetic Transient Simulations", IEEE Transactions on Power Apparatus and Systemps, vol. PAS-101, n.o 1, pp. 147-157, ene. 1982.

[9] J. J. Grainger, W. D. Stevenson, y G.W. Chang. Power System Analysis, McGraw-Hill Education. 2003.

[10]D. P. Kothari y I. J. Nagrath, Modern Power System Analysis. McGraw-Hill Education, 2011.

[11]A. Trujillo, "Análisis de modelos de cálculo de parámetros de líneas de transmisión", Escuela Politécnica Nacional, Quito, Ecuador, 2013.

[12] A. B. Fernandes, W. L. A. Neves, E. G. da Costa, y M. N. Cavalcanti, "The Effect of the Shunt Conductance on Transmission Line Models", 2001.

[13]B. Chiguano, J. Ramírez, F. Quilumba, y C. Gallardo, "Estimación de los Parámetros Eléctricos de un Generador Sincrónico basada en Mediciones de Laboratorio usando Métodos de Optimización No Lineal.", Revista Técnica Energía, vol.15, n.o 2, 2018.

[14]H. W. Dommel, "Digital Computer Solution of Electromagnetic Transients in Single-and Multiphase Networks", IEEE Trans. Power Appar. Syst., vol. PAS-88, n.o 4, pp. 388-399, abr. 1969.

[15] M. Rodas., "Estudio de una línea de transmisión mediante un modelo", Escuela Politécnica Nacional, Quito, Ecuador, 1970. 
[16] J. Horak, "Zero sequence impedance of overhead transmission lines", en 59th Annual Conference for Protective Relay Engineers, College Station, Texas, Estados Unidos de América, 2006.

[17]B. Chiguano, "Estimación de parámetros eléctricos de la máquina sincrónica utilizando MATLABSimulink", Escuela Politécnica Nacional, Quito, Ecuador, 2018.

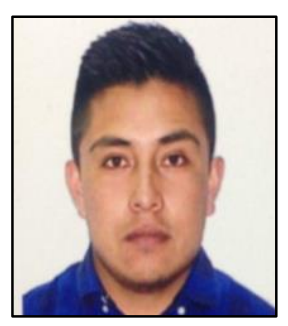

René D. Gualotuña.- Realizó sus estudios primarios en la escuela "Fiscal Mixta José Mejía Lequerica y secundarios en el "Instituto Tecnológico Superior Central Técnico" donde obtuvo el título de Bachiller en Electrónica. Realizó sus estudios universitarios en la Escuela Politécnica Nacional, donde obtuvo el título de Ingeniero Eléctrico en el 2019.

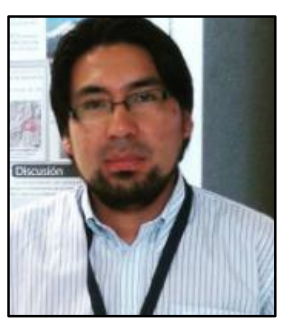

Juan D. Ramírez.- Se graduó como Ingeniero Eléctrico en la Escuela Politécnica Nacional en el 2015. Actualmente está realizando sus estudios de posgrado en Administración de Empresas de los Sectores Estratégicos en la Escuela Politécnica Nacional. Se ha desempeñado como especialista técnico en la construcción de líneas de transmisión de electricidad en CELEC EP TRANSELECTRIC y en la actualidad trabaja como Profesor Ocasional en el Departamento de Energía Eléctrica de la Escuela Politécnica Nacional. Sus áreas de interés incluyen: teorías de sistemas y control aplicadas al sistema eléctrico de potencia, modelación y simulación de sistemas eléctricos, ingeniería de alto voltaje, y operaciones comerciales del sector eléctrico.

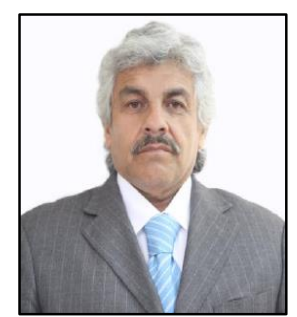

Miguel Á. Lucio.- Nació en la ciudad de Quito- Ecuador el 23 de julio de 1957. Obtuvo el título de Ingeniero Eléctrico con especialidad en Potencia en la Escuela Politécnica Nacional en el año 1981 y su maestría en Gestión de la Energía en la Universidad Técnica de Cotopaxi en el año 2013. Actualmente se desempeña como Docente a Tiempo Completo en el Departamento de Energía Eléctrica de la Escuela Politécnica Nacional. título de Ingeniero Eléctrico. Sus intereses abarcan estudios de Alto Voltaje y Distribución de Energía Eléctrica.

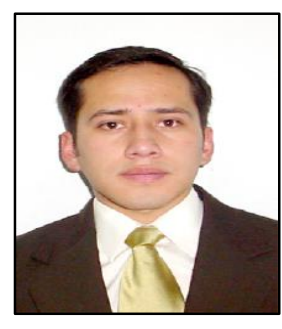

Nelson V. Granda.- Obtuvo el título de Ingeniero Eléctrico en la Escuela Politécnica Nacional en el año 2006 y de Doctor en Ciencias de la Ingeniería Eléctrica en la Universidad Nacional de San Juan (Argentina), en el año 2015. Se ha desempeñado como Ingeniero Eléctrico en varias instituciones del sector eléctrico y petrolero como son el Operador Nacional de Electricidad (CENACE), Petroamazonas EP y CELECEP TRANSELECTRIC.

Actualmente se desempeña como parte del staff docente del Departamento de Energía Eléctrica de la Escuela Politécnica Nacional. Sus áreas de interés son análisis y control de sistemas eléctricos de potencia en tiempo real y aplicaciones de Sistemas de Medición de Área extendida (WAMS) basados en unidades de medición sincrofasorial (PMU).

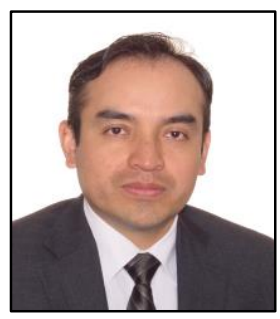

Franklin L. Quilumba.- Obtuvo el título de Ingeniero Eléctrico en la Escuela Politécnica Nacional en Quito, Ecuador, en el 2008. Realizó sus estudios de posgrado en la Universidad de Texas Arlington, en Arlington, Estados Unidos de América, donde obtuvo el grado de Master of Science y el título de Doctor of Philosophy Ph.D., en Ingeniería Eléctrica, en el 2014. Entre 2014 y 2018 fue docente titular en el Departamento de Energía Eléctrica de la Escuela Politécnica Nacional. En la actualidad se desempeña como ingeniero de protecciones de sistemas de transmisión de la compañía Oncor Electric Delivery, Fort Worth, TX, USA. Sus áreas de interés incluyen modelación y análisis de sistemas eléctricos de potencia (SEPs); estabilidad, protección y control de SEPs; planificación de SEPs. El Dr. Quilumba es Ingeniero Profesional Registrado en el Estado de Texas. 